\title{
PRESSURE MAXIMUM BEHAVIOR IN INFLATION OF INCOMPRESSIBLE ELASTIC HOLLOW SPHERES AND CYLINDERS*
}

\author{
By \\ M. M. CARROLL \\ University of California, Berkeley
}

\begin{abstract}
It is shown that realistic material response allows for three qualitatively different types of behavior for inflation of incompressible elastic hollow spheres and stretched cylinders. The pressure may increase monotonically, or it may increase and then decrease, or it may increase, decrease, and then increase again. A simple condition on the material response curve for uniaxial compressive stress is used to classify materials with respect to spherical inflation and to examine which type of behavior will occur for a particular material and initial geometry. Similar results are obtained for inflation of axially stretched hollow cylinders. The results are also applicable to elastic-plastic strain hardening materials.
\end{abstract}

1. Introduction. Problems of finite inflation of hollow spheres and cylinders of homogeneous, isotropic, incompressible solid material have been treated fairly extensively in the literature. These deformations belong to a class of deformations, called controllable or universal, which can be supported in all such materials without application of body force. The discovery of such solutions by Rivlin gave a considerable impetus to the study of nonlinear elasticity. Treatment of problems involving controllable deformations is simplified greatly by the fact that the deformation field is known $a b$ initio. The solution for finite inflation of elastic hollow cylinders, which may also undergo uniform axial stretching, was first given by Rivlin [1], and that for finite inflation of elastic hollow spheres was given by Green and Shield [2].

An unusual feature of these solutions is that the qualitative behavior of the internal pressure may be different for different materials. The pressure may (a) increase monotonically, (b) increase and then decrease, or (c) inciease, decrease, and then increase again, depending on the form of the strain energy function and on the initial geometry. Pressure maximum behavior is observed, for example, in blowing up toy balloons.

* Received November 18, 1985. 
All of these effects are, of course, inherent in the general solutions by Rivlin [1] and Green and Shield [2], and they have been examined in more detail by other authors [3-7]. However, there does not appear to have been a general treatment of the various types of qualitative behavior which are possible, or the development of a set of conditions which would determine, a priori, which type of behavior will occur for a specific material and initial geometry.

These conditions are found in the present paper. For both the spherical and cylindrical problems, it is shown that realistic forms of the strain energy function admit all three types of behavior (a), (b), and (c) above. Materials are classified into three types-A, B, and $\mathrm{C}$-based on a simple condition on the stress/axial strain response for uniaxial compressive stress. Materials of types A and B exhibit behaviors of types (a) and (b), respectively, in spherical inflation. In the case of materials of type $C$, the qualitative behavior depends on the initial geometry; sufficiently thick-walled spheres exhibit behavior of type ( $a$ ) and sufficiently thin-walled spheres exhibit behavior of type (c). For all three types of materials, inflation of a spherical cavity in an infinite medium requires monotonically increasing pressure. Similar results pertain to cylindrical inflation, except that the relevant material response property is that in plane deformation with uniform normal prestretch. Interestingly, the widely used Mooney-Rivlin strain energy function, including the special case of a neo-Hookean material, allows all three types of behavior in spherical inflation but only behavior of type (a) in cylindrical inflation.

The controllable deformations provide closed form solutions not only for elastic materials but also for homogeneous, isotropic, incompressible materials which exhibit inelastic response (Carroll [8,9], Fosdick [10]). Indeed, the solutions presented here pertain equally to elastic-plastic materials (Carroll [11]). Also, because of incompressibility, solutions for internal pressurization are valid also for all-round tension, so that results on pressure maximum behavior can have important implications with regard to void growth in tensile stress fields.

2. Constitutive Equations. The strain energy for a homogeneous, isotropic, incompressible elastic solid material is a function of two invariants

$$
W=W\left(I_{1}, I_{2}\right) \text {, }
$$

with

$$
I_{1}=\operatorname{tr} \mathbf{B}=\lambda_{1}^{2}+\lambda_{2}^{2}+\lambda_{3}^{2}, \quad I_{2}=\operatorname{tr} \mathbf{B}^{-1}=1 / \lambda_{1}^{2}+1 / \lambda_{2}^{2}+1 / \lambda_{3}^{2},
$$

and

$$
\mathbf{B}=\mathbf{V}^{2}=\mathbf{F F}^{T} \text {. }
$$

Here $\mathbf{F}$ and $\mathbf{F}^{T}$ denote the deformation gradient and its transpose, and the stretch tensor $\mathbf{V}$, the deformation tensor $\mathbf{B}$, and its inverse $\mathbf{B}^{-1}$ are positive-definite and symmetric. The principal stretches $\lambda_{i}(i=1,2,3)$ are the principal values of $\mathbf{V}$, and they meet the isochoric condition

$$
\operatorname{det} \mathbf{V}=\lambda_{1} \lambda_{2} \lambda_{3}=1
$$

The Cauchy stress tensor $\mathbf{T}$ is given by

$$
\mathbf{T}=-p \mathbf{1}+2 \frac{\partial W}{\partial I_{1}} \mathbf{B}-2 \frac{\partial W}{\partial I_{2}} \mathbf{B}^{-1},
$$


where $p$ is an unspecified scalar and $\mathbf{1}$ denotes the unit tensor. The principal directions of $\mathbf{T}$ are those of $\mathbf{V}$.

We introduce functions $\hat{W}, \tilde{W}$, and $\bar{W}$, defined by

$$
\begin{aligned}
\hat{W}(\lambda, \mu) & =W\left(\lambda^{2}+1 /\left(\lambda^{2} \mu^{2}\right)+\mu^{2}, 1 / \lambda^{2}+\lambda^{2} \mu^{2}+1 / \mu^{2}\right), \\
\tilde{W}(\lambda) & =\hat{W}(\lambda, 1 / \sqrt{\lambda}), \\
\bar{W}(\lambda) & =\hat{W}(\lambda, 1) .
\end{aligned}
$$

The function $\hat{W}$ describes the general response in terms of two independent principal stretches, while $\tilde{W}$ and $\bar{W}$ describe the response in axisymmetric and plane deformations, respectively. The principal stress differences, for principal stretches

$$
\lambda_{i}=(\lambda, 1 /(\lambda \mu), \mu)
$$

are given by

$$
t_{1}-t_{2}=\lambda \partial \hat{W} / \partial \lambda, \quad t_{3}-t_{2}=\mu \partial \hat{W} / \partial \mu .
$$

We will be particularly interested in the principal stress difference

$$
\sigma=t_{2}-t_{1}
$$

and we find that this is given, for general deformations and for axisymmetric and plane deformations, respectively, by

$$
\sigma=\left\{\begin{array}{l}
-\lambda \partial \hat{W} / \partial \lambda=\hat{\sigma}(\lambda, \mu), \\
-\lambda d \tilde{W} / d \lambda=\tilde{\sigma}(\lambda), \\
-\lambda d \bar{W} / d \lambda=\bar{\sigma}(\lambda) .
\end{array}\right.
$$

The function $\tilde{\sigma}$ relates the compressive stress to the axial stretch in a uniaxial compressive stress test. Also, $\tilde{\sigma}(\lambda)$ is the tensile stress which corresponds to an in-plane stretch $1 / \sqrt{\lambda}$ in axisymmetric plane stress. Since an isochoric plane deformation is a pure shear, the function $\bar{\sigma}$ is related to the generalized shear modulus.

\section{Inflation of a hollow sphere}

Basic equations. A radially symmetric, isochoric deformation of a hollow sphere is described by

$$
r^{3}-r_{0}^{3}=a^{3}-a_{0}^{3}, \quad \theta=\theta_{0}, \quad \phi=\phi_{0},
$$

where $(r, \theta, \phi)$ are spherical polar coordinates of a typical particle, $a$ is the radius of the inner boundary, and the subscript 0 denotes initial value. The deformation (3.1) may be described in terms of a nondimensional parameter $\alpha$, with initial value $\alpha_{0}$, which is a measure of the porosity of the sphere and is defined by

$$
\alpha=b^{3} /\left(b^{3}-a^{3}\right), \quad \alpha_{0}=b_{0}^{3} /\left(b_{0}^{3}-a_{0}^{3}\right),
$$

where $b$ is the outer radius. As the sphere inflates, the porosity $\alpha$ increases and the local deformation is a radial contraction, with stretch $\lambda$ given by

$$
\lambda=\frac{d r}{d r_{0}}=\frac{r_{0}^{2}}{r^{2}}=\left\{1-\frac{a^{3}-a_{0}^{3}}{r^{3}}\right\}^{2 / 3},
$$


and with tangential and azimuthal stretches $1 / \sqrt{\lambda}$. Equations (3.1), (3.2), and (3.3) imply

$$
\frac{a^{3}}{a_{0}^{3}}=\frac{\alpha-1}{\alpha_{0}-1}, \quad \frac{b^{3}}{b_{0}^{3}}=\frac{\alpha}{\alpha_{0}}, \quad \lambda^{3 / 2}=1-\frac{a_{0}^{3}\left(\alpha-\alpha_{0}\right)}{r^{3}\left(\alpha_{0}-1\right)} .
$$

The radial equation of equilibrium is

$$
\frac{d T_{r r}}{d r}+\frac{2}{r}\left(T_{r r}-T_{\theta \theta}\right)=0
$$

and the boundary conditions, in the case of internal pressurization, are

$$
T_{r r}=-P \quad \text { at } \quad r=a, \quad T_{r r}=0 \quad \text { at } \quad r=b .
$$

Equations (2.9) and (2.11) give the principal stress difference $T_{\theta \theta}-T_{r r}$ as

$$
T_{\theta \theta}-T_{r r}=\tilde{\sigma}(\lambda)
$$

with $\lambda$ given by (3.4) ${ }_{3}$. Equations (3.5)-(3.7) give

$$
P=2 \int_{a}^{b} \tilde{\sigma}(\lambda) \frac{d r}{r} .
$$

A change of variable of integration gives

$$
P=\frac{2}{3} \int_{x_{a}}^{x_{b}} \tilde{\sigma}\left(x^{2 / 3}\right) \frac{d x}{1-x},
$$

with

$$
x=\lambda^{3 / 2}=1-\frac{a^{3}-a_{0}^{3}}{r^{3}}=1-\frac{a_{0}^{3}\left(\alpha-\alpha_{0}\right)}{r^{3}\left(\alpha_{0}-1\right)}
$$

and

$$
x_{a}=\frac{a_{0}^{3}}{a^{3}}=\frac{\alpha_{0}-1}{\alpha-1}, \quad x_{b}=\frac{b_{0}^{3}}{b^{3}}=\frac{\alpha_{0}}{\alpha} .
$$

Condition for stationary values of $P$. Differentiation of Eq. (3.9), using Leibnitz' rule, gives

$$
\frac{d P}{d \alpha}=\frac{2}{3\left(\alpha-\alpha_{0}\right)}\left[\frac{\alpha_{0}-1}{\alpha-1} \tilde{\sigma}\left\{\left(\frac{\alpha_{0}-1}{\alpha-1}\right)^{2 / 3}\right\}-\frac{\alpha_{0}}{\alpha} \tilde{\sigma}\left\{\left(\frac{\alpha_{0}}{\alpha}\right)^{2 / 3}\right\}\right] .
$$

It is convenient to introduce a response function $\tilde{g}$, defined by

$$
\tilde{g}(x)=x \tilde{\sigma}\left(x^{2 / 3}\right),
$$

in terms of which Eq. (3.12) becomes

$$
\frac{d P}{d \alpha}=\frac{2}{3\left(\alpha-\alpha_{0}\right)}\left[\tilde{g}\left(\frac{\alpha_{0}-1}{\alpha-1}\right)-\tilde{g}\left(\frac{\alpha_{0}}{\alpha}\right)\right] .
$$

The condition for a stationary value of the applied pressure $P$ is

$$
\tilde{g}\left(\frac{\alpha_{0}-1}{\alpha-1}\right)=\tilde{g}\left(\frac{\alpha_{0}}{\alpha}\right) .
$$

This condition may also be written as

$$
\lambda_{a}^{3 / 2} \sigma_{a}=\lambda_{b}^{3 / 2} \sigma_{b}
$$


where $\lambda_{a}, \lambda_{b}$ and $\sigma_{a}, \sigma_{b}$ denote the values of the radial stretch $\lambda$ and the compressive radial stress $\sigma=T_{\theta \theta}-T_{r r}$ at $r=a$ and $r=b$. Differentiation of Eq. (3.14) and use of Eq. (3.15) gives an expression for $d^{2} P / d \alpha^{2}$ at a stationary point;

$$
\left(\frac{d^{2} P}{d \alpha^{2}}\right) *=\frac{2}{3\left(\alpha-\alpha_{0}\right)}\left\{-\frac{\alpha_{0}-1}{(\alpha-1)^{2}} \tilde{g}^{\prime}\left(\frac{\alpha_{0}-1}{\alpha-1}\right)+\frac{\alpha_{0}}{\alpha^{2}} \tilde{g}^{\prime}\left(\frac{\alpha_{0}}{\alpha}\right)\right\} .
$$

It follows from Eqs. (3.15) and (3.17) that the qualitative behavior of the pressure $P$ in spherical inflation is determined by the form of the function $\tilde{g}$ on the interval $(0,1)$.

Qualitative behavior of the pressure. We can now describe the qualitative behavior of the pressure during finite inflation of a hollow sphere in terms of the uniaxial compressive stress response of the material. It is convenient to write this response in terms of the compressive logarithmic strain $\varepsilon$, thus

$$
\sigma=\sigma(\varepsilon)=\tilde{\sigma}\left(e^{-\varepsilon}\right), \quad \varepsilon=-\ln \lambda .
$$

Equations (3.10), (3.13), and (3.18) give

$$
\tilde{g}\left(\lambda^{3 / 2}\right)=g(\varepsilon)=e^{-3 \varepsilon / 2} \sigma(\varepsilon) .
$$

The condition (3.15) for a stationary value of the pressure $P$ is that the function $g$ must take the same value at the inner and outer boundaries of the hollow sphere. Thus, the relevant material property is monotonicity, or loss of monotonicity, of the function $g$ on $(0, \infty)$. It follows from Eq. (3.19) that $g$ is monotonic when

$$
d \sigma / d \varepsilon \geqslant \frac{3}{2} \sigma .
$$

Three basic types of compressive uniaxial stress-strain curves are shown in Fig. 1. The behavior of the functions $\tilde{g}\left(x_{a}\right)$ and $\tilde{g}\left(x_{b}\right)$ is shown in Fig. 2 and the corresponding qualitative behavior of the pressure in spherical inflation is shown in Fig. 3.

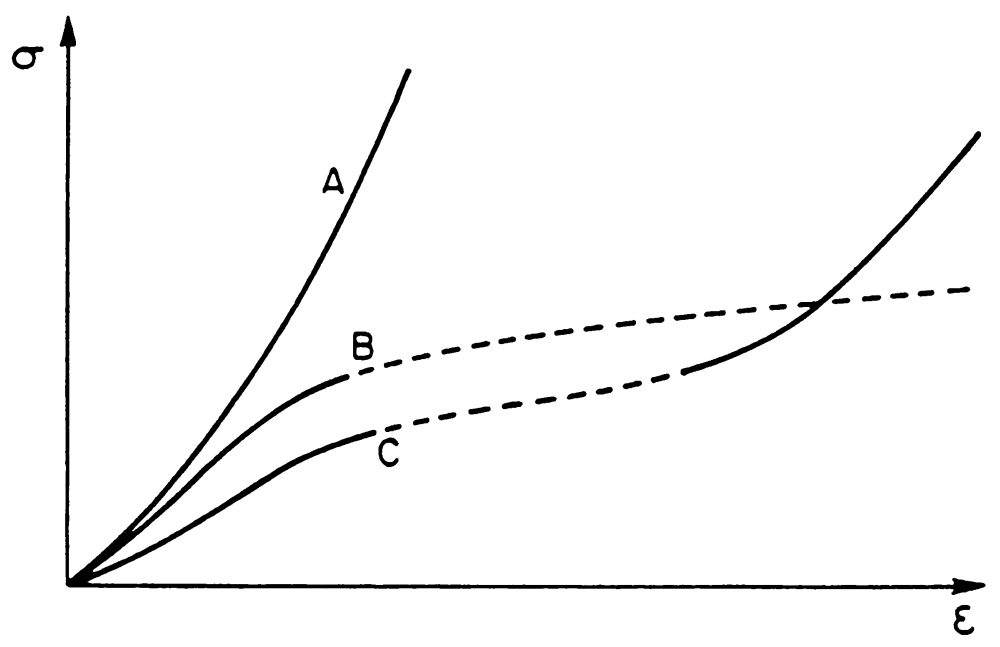

FIG. 1. Uniaxial compressive stress/strain curves. The condition (3.20) is met on solid portions and is not met on dashed portions. 
Material of type A. The condition (3.20) is met over the entire range of compressive axial strain $0 \leqslant \varepsilon<\infty$. It follows that the function $\tilde{g}(x)$ is monotonic for $1 \geqslant x>0$, so that Eq. (3.15) does not have a real root $\alpha^{*}$ with $\alpha_{0}<\alpha^{*}<\infty$ (Fig. 2a). Thus, there are no stationary values of $P$ and the pressure increases monotonically (Fig. 3).

Material of type B. The condition (3.20) is met for $0 \leqslant \varepsilon \leqslant \varepsilon_{1}$ and is not met for $\varepsilon_{1}<\varepsilon<\infty$ (Fig. 1). It follows that the function $\tilde{g}(x)$ has its maximum value in $(0,1)$, at $x_{1}=\exp \left(-3 \varepsilon_{1} / 2\right)$, and so Eq. (3.15) has one real root $\alpha^{*}$, with $\alpha_{0}<\alpha^{*}<\infty$ (Fig. 2b). It is evident also that

$$
\tilde{g}^{\prime}\left(\frac{\alpha_{0}-1}{\alpha^{*}-1}\right)>0, \quad \tilde{g}\left(\frac{\alpha_{0}}{\alpha^{*}}\right)<0
$$

The pressure $P$ increases monotonically to a maximum value $P^{*}$ at porosity $\alpha^{*}$ and decreases monotonically thereafter (Fig. 3). In the special case $\tilde{g}(x)=$ constant for $x \leqslant x_{1}$, the pressure reaches its maximum value at $\alpha^{*}=\alpha_{0} / x_{1}$ and remains constant thereafter.

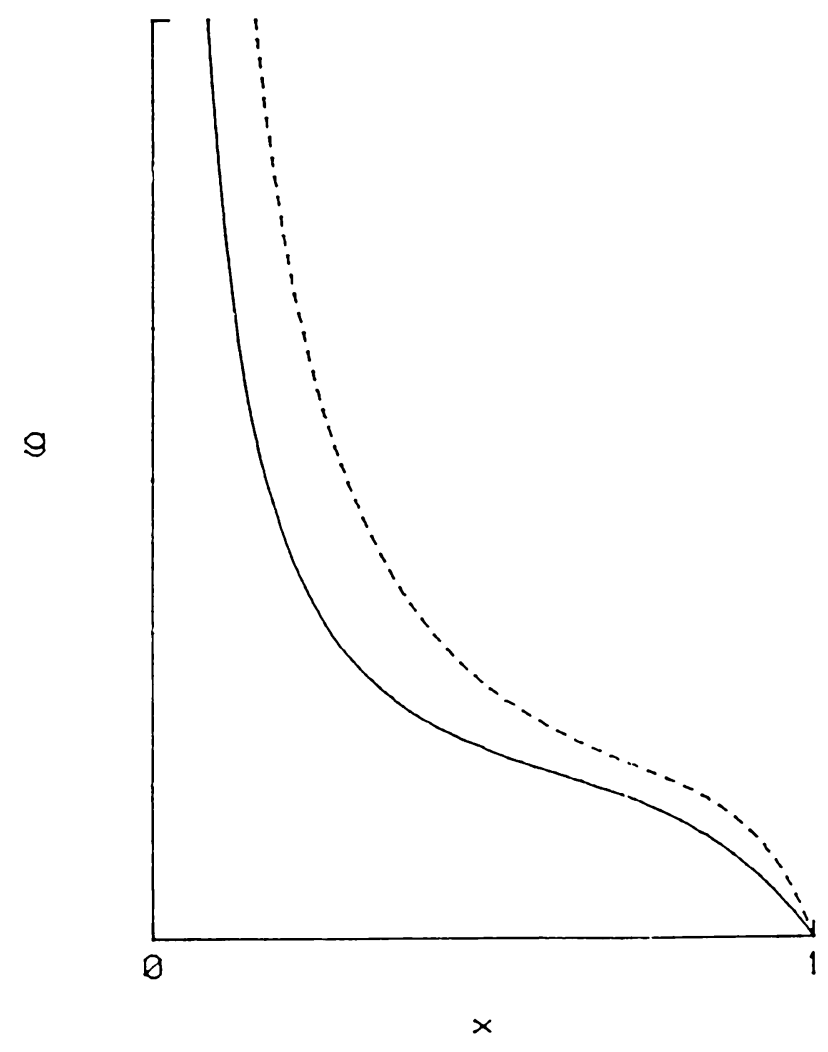

FIG. 2. Qualitative behavior of the function $\tilde{g}(x)$. Solid curves represent $\tilde{g}\left(x_{b}\right)$ and dashed curves represent $\tilde{g}\left(x_{a}\right)$.

(a) Equation (3.15) does not have an admissible root. 


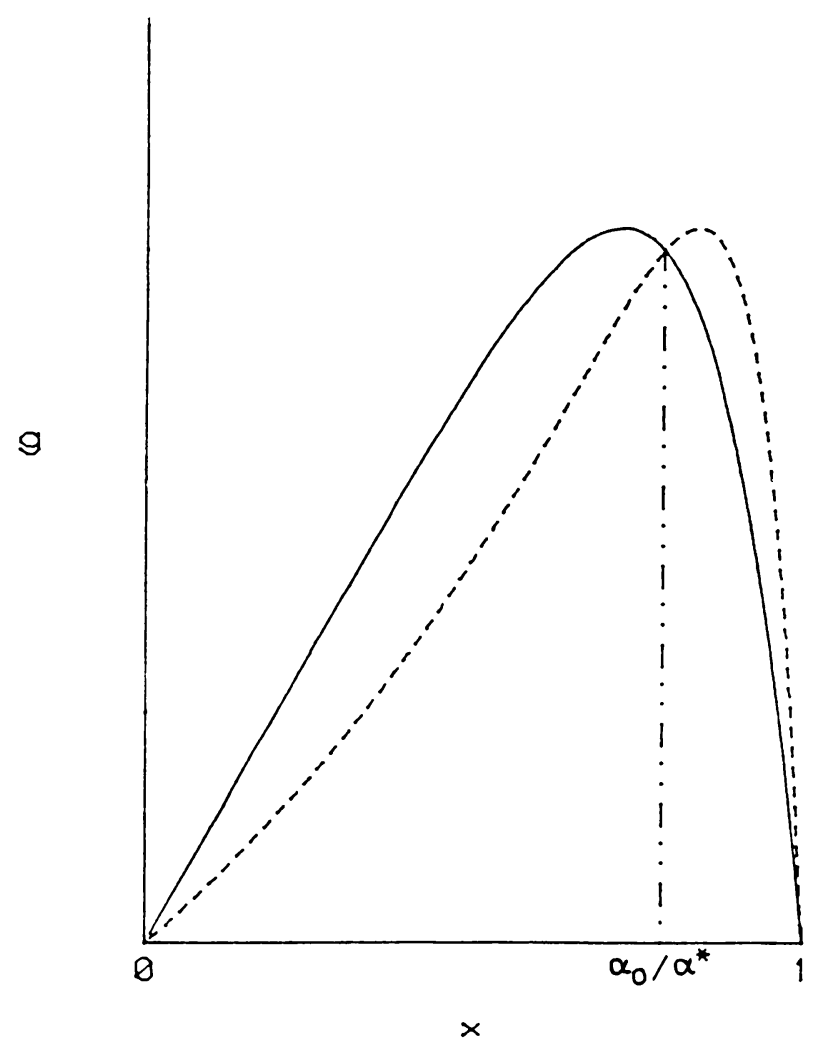

FIG. 2. Qualitative behavior of the function $\tilde{g}(x)$. Solid curves represent $\tilde{g}\left(x_{h}\right)$ and dashed curves represent $\tilde{g}\left(x_{a}\right)$.

(b) Equation (3.15) has one admissible root $\alpha^{*}$.

Material of type $C$. The condition (3.20) is met except on the finite interval $\varepsilon_{1}<\varepsilon<\varepsilon_{2}$ (Fig. 1). The function $\tilde{g}(x)$ has a local maximum and a local minimum on $(0,1)$, at $x_{1}=\exp \left(-3 \varepsilon_{1} / 2\right)$ and $x_{2}=\exp \left(-3 \varepsilon_{2} / 2\right)$, respectively. We observe from Eqs. (3.11) that

$$
x_{a}=\left(1-\beta_{0}\right) x_{b} /\left(1-\beta_{0} x_{b}\right), \quad \beta_{0}=1 / \alpha_{0},
$$

so that the values $x_{a}$ and $x_{b}$ are close for thin-walled spheres (larger values of $\alpha_{0}$ ) and significantly different for thick-walled spheres (smaller values of $\alpha_{0}$ ). Thus, for sufficiently thin-walled spheres, Eq. (3.15) has two admissible roots $\alpha^{*}$ and $\alpha^{* *}$ (Fig. 2c). The pressure $P$ increases monotonically to a local maximum $P^{*}$ at $\alpha^{*}$, decreases to a local minimum $P^{* *}$ at $\alpha^{* *}$, and then increases monotonically (Fig. 3). For sufficiently thick-walled spheres, Eq. (3.15) does not have an admissible root (Fig. $2 \mathrm{~d}$ ) and the pressure $P$ increases monotonically. There is a critical value of $\alpha_{0}$ for which Eq. (3.15) has one admissible repeated root; the pressure $P$ is monotonic but has an inflection point. 


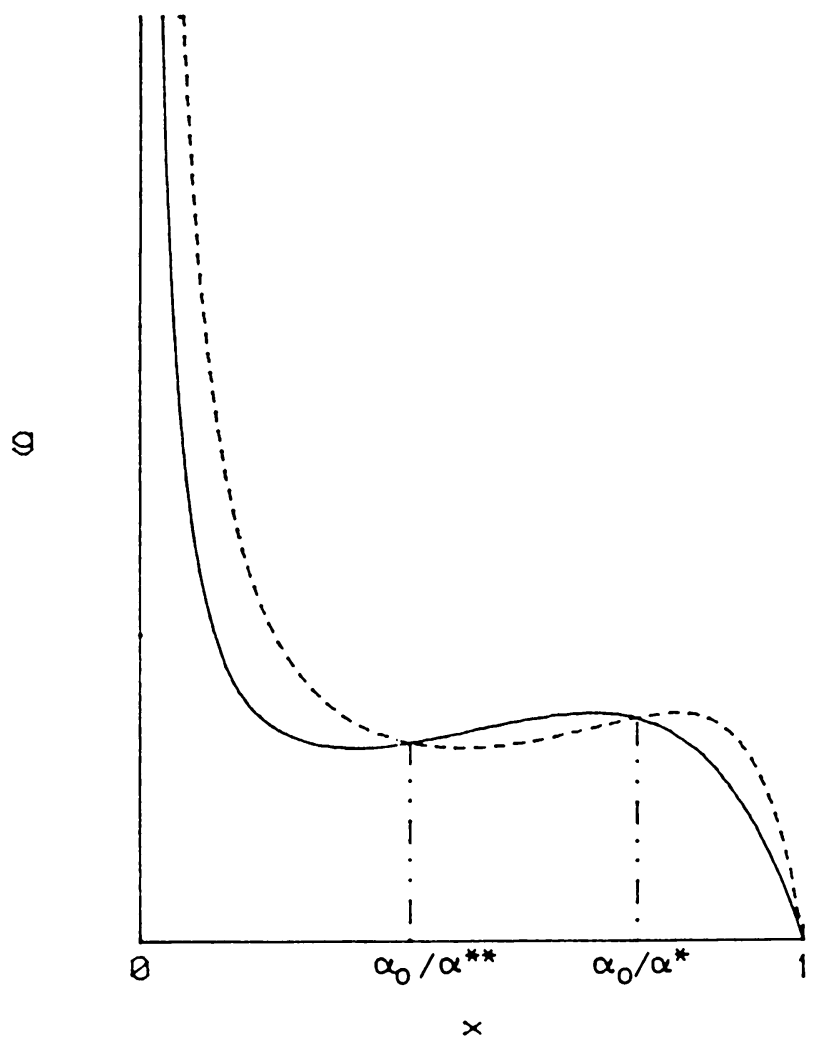

FIG. 2. Qualitative behavior of the function $\tilde{g}(x)$. Solid curves represent $\tilde{g}\left(x_{b}\right)$ and dashed curves represent $\tilde{g}\left(x_{a}\right)$.

(c) Equation (3.15) has two admissible roots $\alpha^{*}$ and $\alpha^{* *}$, for sufficiently large values of $\alpha_{0}$.

The three types of behavior just described do not exhaust the possibilities. It is conceivable, for example, that condition (3.20) might be violated on two finite intervals. However, the compressive uniaxial stress response of most real materials should be type A, $\mathrm{B}$, or $\mathrm{C}$. Thus, we expect that the pressure in spherical inflation will increase monotonically (materials of type A and thick-walled spheres of material of type C), or increase to a maximum value and then decrease (materials of type B), or increase, decrease, and then increase again (thin-walled spheres of material of type $C$ ). While our results are derived in the context of finite elasticity, it is evident that they apply also to inflation of hollow spheres of elastic-plastic strain hardening material, which will generally be of type B or possibly of type C.

Mooney-Rivlin material. The three types of behavior just described can be illustrated by consideration of the Mooney-Rivlin strain energy function

$$
W=C_{1}\left(I_{1}-3\right)+C_{2}\left(I_{2}-3\right) .
$$




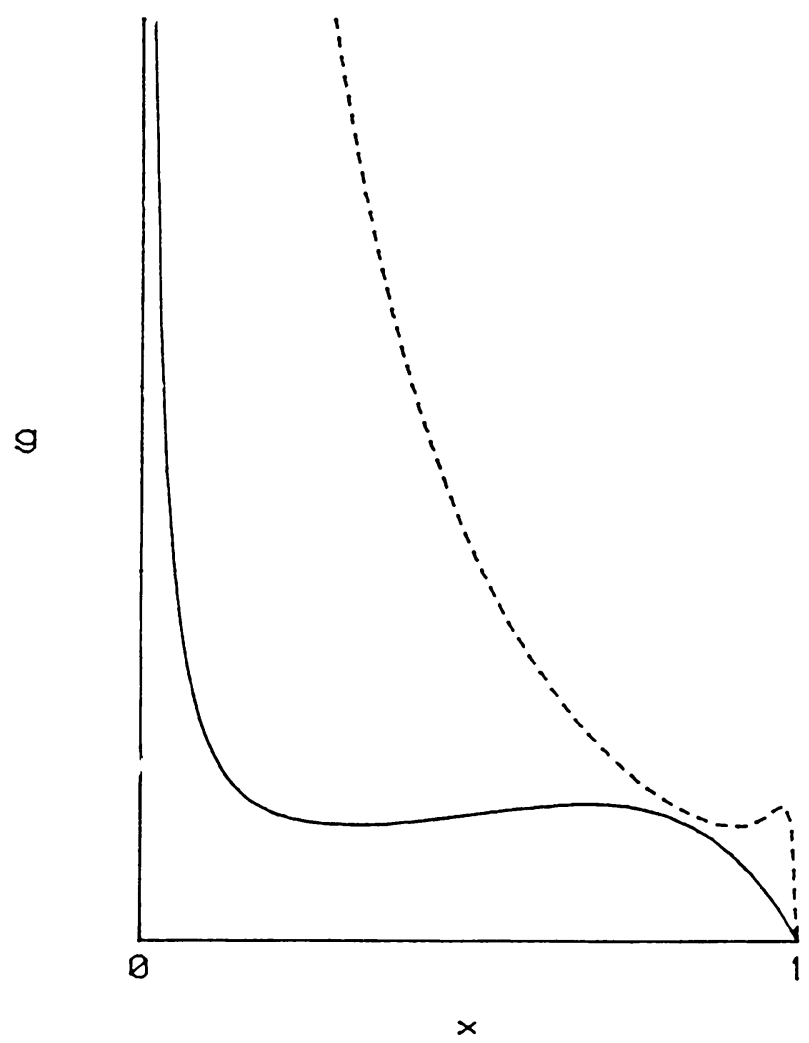

FIG. 2. Qualitative behavior of the function $\tilde{g}(x)$. Solid curves represent $\tilde{g}\left(x_{b}\right)$ and dashed curves represent $\tilde{g}\left(x_{a}\right)$.

(d) Equation (3.15) does not have an admissible root, for sufficiently small values of $\alpha_{0}(>1)$.

The function $\tilde{g}$ may be read off from Eqs. (2.6), (2.10), (3.13), and (3.23), viz.,

$$
\tilde{g}(x)=2 C_{1}\left\{x^{1 / 3}-x^{7 / 3}+\kappa\left(x^{-1 / 3}-x^{5 / 3}\right)\right\}, \quad \kappa=C_{2} / C_{1} .
$$

Differentiation of Eq. (3.24) gives

$$
\tilde{g}^{\prime}(x)=\frac{2}{3} C_{1} x^{-4 / 3}\left\{x^{2 / 3}\left(1-7 x^{2}\right)-\kappa\left(1+5 x^{2}\right)\right\} .
$$

For $\kappa=0, \tilde{g}^{\prime}$ has one zero in $(0,1)$, at $x_{1}=1 / \sqrt{7}$. Consideration of the function

$$
\hat{\kappa}(x)=x^{2 / 3}\left(1-7 x^{2}\right) /\left(1+5 x^{2}\right)
$$

shows that there is a critical value $\kappa_{\mathrm{cr}} \cong 0.2145$, such that the function $\tilde{g}$ has a local maximum and minimum in $(0,1)$ for $0<\kappa<\kappa_{\mathrm{cr}}$ and is monotonic in $(0,1)$ for $\kappa>\kappa_{\mathrm{cr}}{ }^{1}$ Thus, the Mooney-Rivlin strain energy function (3.23) allows all three types of behavior

\footnotetext{
${ }^{1}$ This result is given by Green and Adkins [3] for a spherical membrane.
} 


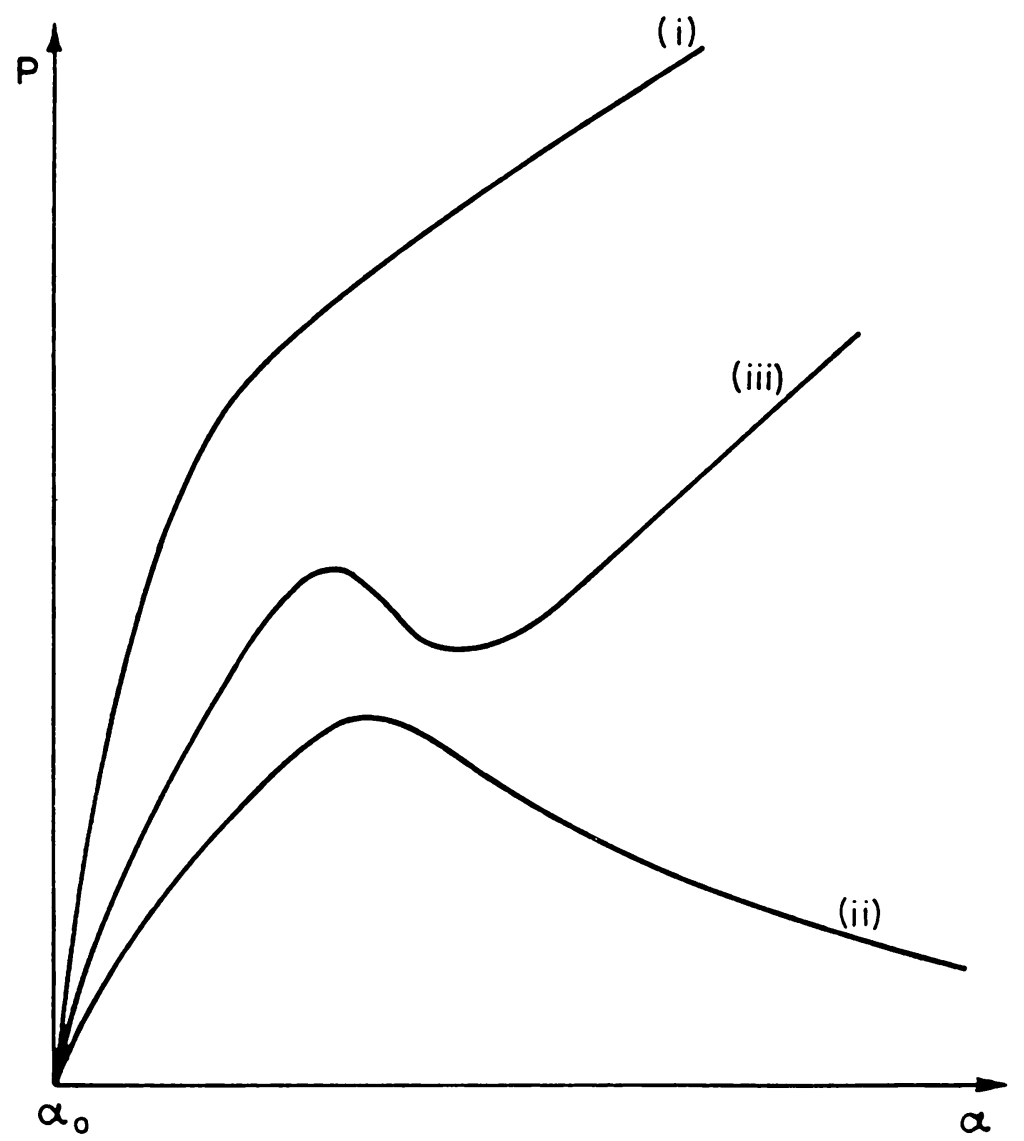

FIG. 3. Qualitative pressure-porosity curves: (i) monotonic, (ii) pressure maximum, (iii) local maximum and minimum.

in spherical inflation. The material is of type $\mathrm{A}$ for $\kappa \geqslant \kappa_{\mathrm{cr}}$, of type $\mathrm{B}$ for $\kappa=0$ (neo-Hookean material), and of type $C$ for $0<\kappa \leqslant \kappa_{\mathrm{cr}}$. In the latter case, the pressure maximum $P^{*}$ and minimum $P^{* *}$, for a sufficiently thin-walled sphere, occur at the roots $\alpha^{*}$ and $\alpha^{* *}$ of

$$
\begin{aligned}
\left\{\left(\frac{\alpha_{0}-1}{\alpha-1}\right)^{1 / 3}+\kappa\left(\frac{\alpha-1}{\alpha_{0}-1}\right)^{1 / 3}\right\}\left\{1-\left(\frac{\alpha_{0}-1}{\alpha-1}\right)^{2}\right\} & \\
= & \left\{\left(\frac{\alpha_{0}}{\alpha}\right)^{1 / 3}+\kappa\left(\frac{\alpha}{\alpha_{0}}\right)^{1 / 3}\right\}\left\{1-\left(\frac{\alpha_{0}}{\alpha}\right)^{2}\right\} .
\end{aligned}
$$

We remark that Ogden [7] has treated strain energy functions which are linear combinations of terms of the form

$$
W=C\left(\lambda_{1}^{m}+\lambda_{2}^{m}+\lambda_{3}^{m}-3\right) .
$$

It is easily seen that Eq. (3.28) defines a material of type B for

$$
-3 / 2<m<3,
$$


and a material of type A otherwise [7]. Suitable combinations of terms of types A and B define materials of type $\mathrm{C}$, as in the Mooney-Rivlin case.

Spherical cavity in an infinite medium. The pressure response for a pressurized spherical cavity, of initial radius $a_{0}$, in a medium of infinite extent is given by

$$
P=\frac{2}{3} \int_{a_{0}^{3} / a^{3}}^{1} \tilde{\sigma}\left(x^{2 / 3}\right) \frac{d x}{1-x} .
$$

Differentiation of Eq. (3.30) gives

$$
\frac{d P}{d a}=\frac{2 a_{0}^{3}}{3 a\left(a^{3}-a_{0}^{3}\right)} \tilde{\boldsymbol{\sigma}}\left(\frac{a_{0}^{2}}{a^{2}}\right)
$$

so that the pressure increases monotonically.

\section{Inflation and stretching of a hollow cylinder}

Basic equations. The deformation

$$
r^{2}-r_{0}^{2} / \mu=a^{2}-a_{0}^{2} / \mu, \quad \theta=\theta_{0}, \quad z=\mu z_{0},
$$

where $(r, \theta, z)$ are cylindrical coordinates and $\mu$ is a positive constant, describes radial inflation and uniform axial stretching of a circular cylindrical tube of initial inner radius $a_{0}$. It is again convenient to introduce a porosity measure $\alpha$, with

$$
\alpha=b^{2} /\left(b^{2}-a^{2}\right), \quad \alpha_{0}=b_{0}^{2} /\left(b_{0}^{2}-a_{0}^{2}\right),
$$

where $b_{0}$ is the initial outer radius. An initial axial stretching

$$
r^{2}=r_{0}^{2} / \mu, \quad \theta=\theta_{0}, \quad z=\mu z_{0}
$$

does not change the porosity, and the subsequent radial inflation, for fixed value of $\mu$, is described by the variable $\alpha$.

The principal stretches are in the coordinate directions and are given by

$$
\lambda_{i}=\left(\frac{d r}{d r_{0}}, \frac{r}{r_{0}}, \frac{d z}{d z_{0}}\right)=\left(\lambda, \frac{1}{\lambda \mu}, \mu\right),
$$

with the radial stretch $\lambda$ given by

$$
\lambda^{2}=\frac{1}{\mu}\left\{1-\frac{\mu a^{2}-a_{0}^{2}}{\mu r^{2}}\right\} .
$$

Equations (4.1), (4.2), and (4.5) imply that

$$
\frac{\mu a^{2}}{a_{0}^{2}}=\frac{\alpha-1}{\alpha_{0}-1}, \quad \frac{\mu b^{2}}{b_{0}^{2}}=\frac{\alpha}{\alpha_{0}}, \quad \lambda^{2}=\frac{1}{\mu}\left\{1-\frac{a_{0}^{2}\left(\alpha-\alpha_{0}\right)}{\mu r^{2}\left(\alpha_{0}-1\right)}\right\} .
$$

The radial equation of equilibrium is

$$
\frac{d T_{r r}}{d r}+\frac{1}{r}\left(T_{r r}-T_{\theta \theta}\right)=0
$$

and the boundary conditions, for internal pressurization of the tube, are

$$
T_{r r}=-P \quad \text { at } \quad r=a, \quad T_{r r}=0 \quad \text { at } \quad r=b .
$$


It follows from Eq. (4.4) that

$$
T_{\theta \theta}-T_{r r}=\hat{\sigma}(\lambda, \mu)
$$

with the function $\hat{\sigma}$ defined by Eqs. (2.6) $)_{1}$ and (2.10) $)_{1}$. Equations (4.7)-(4.9) give

$$
P=\int_{a}^{b} \hat{\sigma}(\lambda, \mu) \frac{d r}{r},
$$

and a change of variable of integration gives

$$
P=\frac{1}{2} \int_{x_{a}}^{x_{b}} \hat{\sigma}\left\{\left(\frac{x}{\mu}\right)^{1 / 2}, \mu\right\} \frac{d x}{1-x},
$$

with

$$
x=\mu \lambda^{2}, \quad x_{a}=\frac{a_{0}^{2}}{\mu a^{2}}=\frac{\alpha_{0}-1}{\alpha-1}, \quad x_{b}=\frac{b_{0}^{2}}{\mu b^{2}}=\frac{\alpha_{0}}{\alpha} .
$$

Equation (4.11) gives the pressure as a function of the kinematical variable $\alpha$ for inflation of a hollow circular cylinder with axial prestretch. In the special case of inflation without prestretching $(\mu=1)$, it may be written as

$$
P=\frac{1}{2} \int_{x_{u}}^{x_{b}} \bar{\sigma}\left(x^{1 / 2}\right) \frac{d x}{1-x},
$$

where $\bar{\sigma}$ is the plane strain response function defined in Eq. (2.10).

Condition for stationary values of $P$. Differentiation of Eq. (4.1) gives

$$
\frac{d P}{d \alpha}=\frac{1}{2\left(\alpha-\alpha_{0}\right)}\left[\frac{\alpha_{0}-1}{\alpha-1} \hat{\sigma}\left\{\left(\frac{\alpha_{0}-1}{\mu(\alpha-1)}\right)^{1 / 2}, \mu\right\}-\frac{\alpha_{0}}{\alpha} \hat{\sigma}\left\{\left(\frac{\alpha_{0}}{\mu \alpha}\right)^{1 / 2}, \mu\right\}\right] .
$$

Introducing a response function $h$, defined by

$$
h\left(\lambda^{2}, \mu\right)=\lambda^{2} \hat{\boldsymbol{\sigma}}(\lambda, \mu),
$$

gives the condition for stationary values of the applied pressure $P$ in the form

$$
h\left(\frac{\alpha_{0}-1}{\mu(\alpha-1)}, \mu\right)=h\left(\frac{\alpha_{0}}{\mu \alpha}, \mu\right) .
$$

This condition may also be written as

$$
\lambda_{a}^{2} \sigma_{a}=\lambda_{b}^{2} \sigma_{b} .
$$

where $\lambda_{a}$ and $\lambda_{b}$ denote radial stretches and $\sigma_{a}$ and $\sigma_{b}$ denote principal stress differences $\sigma=T_{\theta \theta}-T_{r r}$, evaluated at the inner and outer boundaries $r=a$ and $r=b$.

The condition (4.17) is analogous to the condition (3.15) for spherical inflation. A similar analysis shows that the qualitative behavior in cylindrical inflation depends on monotonicity of the function $h\left(\lambda^{2}, \mu\right)$ on the interval $0<\lambda<1 / \sqrt{\mu}$. This function may (A) be monotonic, (B) have a local maximum, or (C) have a local maximum and minimum. Correspondingly, the pressure in cylindrical inflation may increase monotonically (type $\mathrm{A}$, or type $\mathrm{C}$ for thick-walled cylinders), or it may increase to a maximum value $P^{*}$ and then decrease (type $\mathrm{B}$ ), or it may increase to $P^{*}$, decrease to $P^{* *}$, and then increase again (type $\mathrm{C}$ for thin-walled cylinders). The axial prestretch provides an 
interesting additional feature. In particular, it expands or contracts the range of relevant values of the radial stretch $(0<\lambda \leqslant 1 / \sqrt{\mu})$ depending on whether the prestretch is compressive $(\mu<1)$ or tensile $(\mu>1)$.

Special materials. For the strain energy function in Eq. (3.28), i.e.,

$$
W=C\left(\lambda_{1}^{m}+\lambda_{2}^{m}+\lambda_{3}^{m}-3\right) \text {, }
$$

Eq. (2.10) gives

$$
\hat{\sigma}(\lambda, \mu)=m C\left(1 /\left(\lambda^{m} \mu^{m}\right)-\lambda^{m}\right)
$$

and Eqs. (4.15) and (4.18) give

$$
h\left(\lambda^{2}, \mu\right)=m C\left(\mu^{-m} \lambda^{2-m}-\lambda^{2+m}\right) .
$$

The behavior of such a material in cylindrical inflation is of type B for

$$
-2<m<2,
$$

and otherwise is of type A. In particular, neo-Hookean and Mooney-Rivlin materials do not have a pressure maximum instability in cylindrical inflation. For $|m|<2$, the porosity $\alpha^{*}$, at which the pressure attains a maximum, is a root of

$$
\left\{\frac{\alpha_{0}-1}{\mu(\alpha-1)}\right\}^{1+m / 2}-\left\{\frac{\alpha_{0}-1}{\mu(\alpha-1)}\right\}^{1-m / 2}=\left(\frac{\alpha_{0}}{\mu \alpha}\right)^{1+m / 2}-\left(\frac{\alpha_{0}}{\mu \alpha}\right)^{1-m / 2},
$$

which gives the same value for $m$ and $-m$.

For a material with strain energy function

$$
W=M\left(\lambda_{1}^{m}+\lambda_{2}^{m}+\lambda_{3}^{m}-3\right)+N\left(\lambda_{1}^{n}+\lambda_{2}^{n}+\lambda_{3}^{n}-3\right),
$$

where $M, N, m$, and $n$ are constants, with $|m|<2$ and $|n|>2$, Eq. (4.19) leads to

$$
h\left(\lambda^{2}, \mu\right)=m M\left(\mu^{-m} \lambda^{2-m}-\lambda^{2+m}\right)+n N\left(\mu^{-n} \lambda^{2-n}-\lambda^{2+n}\right) .
$$

Analysis similar to that for a Mooney-Rivlin material in spherical inflation shows that this material exhibits behavior of type $\mathrm{C}$ in cylindrical inflation for sufficiently small values of the ratio $N / M$.

Cylindrical cavity in an infinite medium. For pressurization of a cylindrical cavity of radius $a_{0}$ in a medium of infinite extent, with axial stretch $\mu$, the pressure is given by

$$
P=\frac{1}{2} \int_{a_{0}^{2} / \mu a^{2}}^{1} \hat{\sigma}\left\{(x / \mu)^{1 / 2}, \mu\right\} \frac{d x}{1-x} .
$$

Differentiation of Eq. (4.24) gives

$$
\frac{d P}{d \alpha}=\frac{a_{0}^{2}}{a\left(\mu a^{2}-a_{0}^{2}\right)} \hat{\boldsymbol{\sigma}}\left(\frac{a_{0}}{\mu a}, 1\right),
$$

so that the pressure increases monotonically.

Acknowledgment. This work was supported in part by a contribution from the Shell Companies Foundation in support of a Shell Distinguished Chair at the University of California, Berkeley, and in part by Grant MEA-820534 from the National Science Foundation (Solid Mechanics Program) to the University of California, Berkeley. 


\section{REFERENCES}

[1] R. S. Rivlin, Large elastic deformations of isotropic materials. VI. Further results in the theory of torsion, shear and flexure, Philos. Trans. Roy. Soc. London A242, 173 (1949)

[2] A. E. Green and R. T. Shield, Finite elastic deformations in incompressible isotropic bodies, Proc. Roy. Soc. London A202, 407 (1950)

[3] A. E. Green and J. E. Adkins, Large elastic deformations, Clarendon, Oxford, 1970

[4] H. Alexander, Tensile instability of initially spherical balloons, Internat. J. Eng. Sci. 9, 151 (1971)

[5] R. T. Shield, On the stability of finitely deformed elastic membranes. II: Stability of inflated cylindrical and spherical membranes, Z. Angew. Math. Phys. 23, 16 (1972)

[6] A. Needleman, Inflation of spherical rubber balloons, Internat. J. Solids Struct. 13, 409 (1977)

[7] R. W. Ogden, in Mechanics of solids, the Rodney Hill Anniversary Volume (H. G. Hopkins and M. J. Sewell, eds.), Pergamon, Oxford, 1982

[8] M. M. Carroll, Controllable deformations of incompressible simple materials, Internat. J. Eng. Sci. 5, 515 (1967)

[9] M. M. Carroll, Finite deformations of incompressible simple solids I. Isotropic solids, Quart. J. Mech. Appl. Math. 21, 147 (1968)

[10] R. J. Fosdick, Dynamically possible motions of incompressible, isotropic, simple materials, Arch. Rat. Mech. Anal. 29, 272 (1968)

[11] M. M. Carroll, Radial expansion of hollow spheres of elastic-plastic hardening material, Internat. J. Solids Struct. 21, 645 (1985) 